\title{
Poster: Answering the Requirements Traceability Questions
}

\author{
Arushi Gupta \\ Department of EECS \\ University of Cincinnati \\ Cincinnati, Ohio \\ gupta2ai@mail.uc.edu \\ Nan Niu \\ Department of EECS \\ University of Cincinnati \\ Cincinnati, Ohio \\ nan.niu@uc.edu
}

\author{
Wentao Wang \\ Department of EECS \\ University of Cincinnati \\ Cincinnati, Ohio \\ wang2wt@mail.uc.edu \\ Juha Savolainen \\ Software and Control R\&D \\ Danfoss Drives A/S \\ Gråsten, Denmark \\ juhaerik.savolainen@danfoss.com
}

\begin{abstract}
To understand requirements traceability in practice, we present a preliminary study of identifying questions from requirements repositories and examining their answering status. Investigating four open-source projects results in 733 requirements questions, among which $43 \%$ were answered successfully, $35 \%$ were answered unsuccessfully, and $22 \%$ were not answered at all. We evaluate the accuracy of using a state-of-the-art natural language processing tool to identify the requirements questions and illuminate automated ways to classify their answering status.
\end{abstract}

\section{CCS CONCEPTS}

- Software and its engineering $\rightarrow$ Requirements analysis; Traceability;

\section{KEYWORDS}

Traceability, requirements questions, answering status

\section{ACM Reference Format:}

Arushi Gupta, Wentao Wang, Nan Niu, and Juha Savolainen. 2018. Poster: Answering the Requirements Traceability Questions. In ICSE'18 Companion: 40th International Conference on Software Engineering Companion, May 27-June 3, 2018, Gothenburg, Sweden. ACM, New York, NY, USA, 2 pages. https://doi.org/10.1145/3183440.3195049

\section{INTRODUCTION}

The requirements traceability problem refers to the inability to describe and follow the life of a requirement [2]. What specific questions do practitioners actually ask regarding the life of a requirement? How many are successfully answered? What makes the remaining questions difficult to answer successfully or unanswered at all? These illustrate the motivations behind our research.

Permission to make digital or hard copies of part or all of this work for personal or classroom use is granted without fee provided that copies are not made or distributed for profit or commercial advantage and that copies bear this notice and the full citation on the first page. Copyrights for third-party components of this work must be honored. For all other uses, contact the owner/author(s).

ICSE'18 Companion, May 27-June 3, 2018, Gothenburg, Sweden

(c) 2018 Copyright held by the owner/author(s).

ACM ISBN 978-1-4503-5663-3/18/05.

https://doi.org/10.1145/3183440.3195049
Malviya and her colleagues recently conducted an online survey with requirements professionals in the IT industry [6]. Their analysis of 29 survey responses grouped a total of 159 natural language queries into 9 purpose categories. Building on the previous research, we carried out a study to identify the questions that software practitioners asked in the requirements repositories. Table 1 lists the four open-source projects of our study. These projects tackle problems in different domains where different concerns such as security [13] and dependability [16] are important. For each project, we extracted the data from its initial release to its latest stable release. We also balanced factors such as group size $[1]$ and release cycle $[8,14]$ in choosing the projects of Table 1 .

In order to identify requirements traceability questions, it is crucial to recognize where such questions are likely to be recorded. The literature suggests that issue trackers are essential for opensource projects to manage requirements [9]. Common to all the 4 projects of Table 1 is the use of JIRA for requirements management. We relied on the issue type defined in each of the 4 projects to recognize requirements. The widely adopted requirements types included "feature request" and "enhancement", whereas "epic" and "story" $[4,11]$ reflected these projects' agile nature.

Our identification of requirements question from the JIRA repositories was performed at the comment level by leveraging the Stanford CoreNLP toolkit [7]. We removed duplicates (e.g., a comment reply repeated the content that had already been posted before) and filtered out three kinds of irrelevant information: XML, URL, and source code. The comments containing any question recognized by the Stanford CoreNLP formed the result set $Q$.

\section{RESULTS AND ANALYSIS}

We evaluate the results $Q$ by comparing them with the manually created answer sets of the 4 projects. These comparisons are summarized in Table 2. For each open-source project of our study, two researchers built their answer sets individually and reached a substantial degree of agreement: average Cohen's kappa $=0.67$ on requirements traceability questions over 4 projects. The discrepancies were resolved in a joint meeting between the researchers.

Recent work by Liu et al. [5] investigated supervised learning methods for automatically detecting questions in microblogs. Their experiment with 8,465 Sina microblogs (1,278 questions) showed that the combination of lexical, syntax, and contextual features 
Table 1: Four open-source projects from which requirements questions are uncovered.

\begin{tabular}{c||ccc|cccc}
\hline \multirow{2}{*}{ Project } & \multicolumn{3}{c|}{ Characteristics } & \multicolumn{4}{c}{ Statistics } \\
\cline { 2 - 9 } & domain & $\begin{array}{c}\text { written } \\
\text { in }\end{array}$ & $\begin{array}{c}\text { initial } \\
\text { release }\end{array}$ & $\begin{array}{c}\text { \# of days } \\
\text { per release }\end{array}$ & $\begin{array}{c}\text { \# of } \\
\text { req.s }\end{array}$ & $\begin{array}{c}\text { \# of stakehold } \\
\text {-ers per req. }\end{array}$ & $\begin{array}{c}\text { \# of code } \\
\text { files per req. }\end{array}$ \\
\hline \hline AIRFLOW & workflow execution & Python & Oct 16, 2014 & 30.42 & 629 & 2.11 & 11.76 \\
\hline ANY23 & RDF data extraction & Java & July 16, 2012 & 139.52 & 182 & 2.14 & 20.33 \\
\hline DROOLS & business rules & Java & Nov 13, 2012 & 29.36 & 486 & 1.78 & 31.26 \\
\hline JBTM & business process & Java, C++ & Dec 5, 2005 & 21.23 & 1575 & 1.63 & 32.37 \\
\hline
\end{tabular}

Table 2: Accuracy of requirements questions identification.

\begin{tabular}{c||cc|ccc}
\hline Project & $\begin{array}{c}\mid \text { Answer } \\
\text { Set } \mid\end{array}$ & $|Q|$ & Recall & Precision & $F_{1}$ \\
\hline AIRFLOW & 145 & 161 & 0.76 & 0.68 & 0.72 \\
ANY23 & 98 & 93 & 0.69 & 0.73 & 0.71 \\
DROOLS & 96 & 112 & 0.81 & 0.70 & 0.75 \\
JBTM & 394 & 471 & 0.85 & 0.71 & 0.77 \\
\hline
\end{tabular}

achieved the best performance: Recall $=0.73$, Precision $=0.63$, and $F_{1}=0.68$. Our results presented in Table 2 are comparable to this performance level, though our algorithm is unsupervised and mines questions from requirements repositories.

The following conversations extracted from [15] show a false negative (Tom's question beginning with "not sure ...") and a false positive (Tom's suggestion starting with "May I suggest ..."). Possible improvements include semantic parsing beyond the comment level and recognizing project-specific terms and concepts [10].

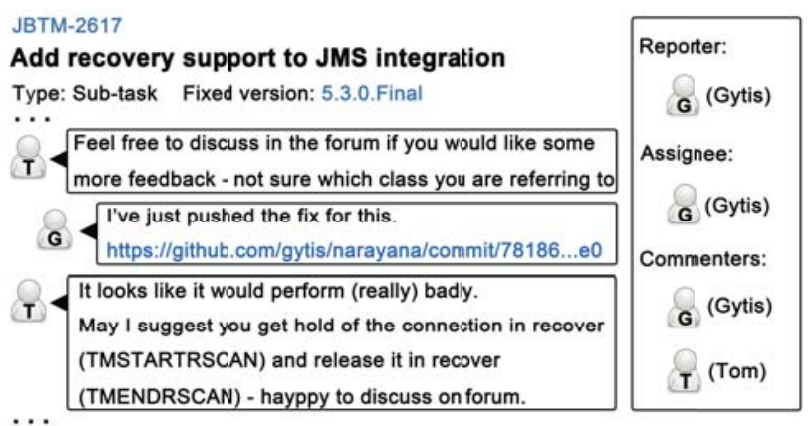

\section{SUMMARY}

The answering status of the 733 requirements questions was analyzed manually and the classification results are shown in Figure 1. Based on our observations, automated methods of classifying the question's answering status could be developed according to features like how requirements traceability is used [12] and whether the diverse chunks of information are complementary [3].

Understanding practitioner questions helps to keep research grounded. We have presented our preliminary results of mining requirements repositories so as to identify questions and to classify whether and how well the questions are answered. Our ongoing work focuses on developing enhanced tools to further automation.

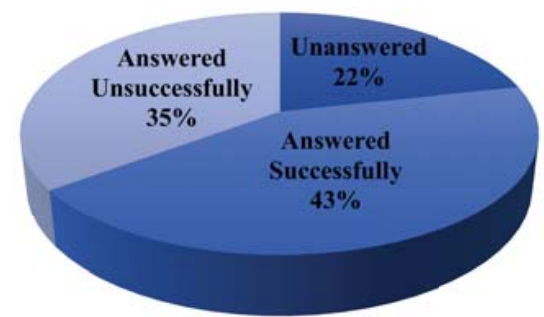

Figure 1: Classifying the 733 requirements questions.

\section{ACKNOWLEDGMENTS}

The work is funded by the U.S. NSF Grant CCF-1350487.

\section{REFERENCES}

[1] T. Bhowmik, N. Niu, W. Wang, J.-R. C. Cheng, L. Li, and X. Cao. Optimal group size for software change tasks: a social information foraging perspective. IEEE Transactions on Cybernetics, 46(8):1784-1795, August 2016.

[2] O. Gotel and A. Finkelstein. An analysis of the requirements traceability problem. In ICRE, pages 94-101, Colorado Springs, CO, USA, April 1994.

[3] X. Jin, N. Niu, and M. Wagner. On the impact of social network information diversity on end-user programming productivity: a foraging-theoretic study. In SSE, pages 15-21, Seattle, WA, USA, November 2016.

[4] C. Khatwani, X. Jin, N. Niu, A. Koshoffer, L. Newman, and J. Savolainen. Advancing viewpoint merging in requirements engineering: a theoretical replication and explanatory study. Requirements Engineering, 22(3):317-338, September 2017.

[5] X. Liu, R. Xie, C. Lin, and L. Cao. Question microblog identification and answer recommendation. Multimedia Systems, 22(4):487-496, July 2016.

[6] S. Malviya, M. Vierhauser, J. Cleland-Huang, and S. Ghaisas. What questions do requirements engineers ask? In $R E$, pages 100-109, Lisbon, Portugal, September 2017.

[7] C. D. Manning, M. Surdeanu, J. Bauer, J. R. Finkel, S. Bethard, and D. McClosky. The Stanford CoreNLP natural language processing toolkit. In ACL, pages 55-60, Baltimore, MD, USA, June 2014.

[8] N. Niu. RE in the age of continuous deployment. In RE, pages 568-569, Lisbon, Portugal, September 2017.

[9] N. Niu, T. Bhowmik, H. Liu, and Z. Niu. Traceability-enabled refactoring for managing just-in-time requirements. In $R E$, pages 133-142, Karlskrona, Sweden, August 2014.

[10] N. Niu and S. Easterbrook: Extracting and modeling product line functional requirements. In $R E$, pages 155-164, Barcelona, Spain, September 2008.

[11] N. Niu, A. Koshoffer, L. Newman, C. Khatwani, C. Samarasinghe, and J. Savolainen Advancing repeated research in requirements engineering: a theoretical replication of viewpoint merging. In $R E$, pages 186-195, Beijing, China, Sept 2016.

[12] N. Niu, W. Wang, and A. Gupta. Gray links in the use of requirements traceability. In FSE, pages 384-395, Seattle, WA, USA, November 2016.

[13] N. Niu, Y. Yu, B. González-Baixauli, N. A. Ernst, J. C. S. do Prado Leite, and J. Mylopoulos. Aspects across software life cycle: a goal-driven approach. Transactions on Aspect-Oriented Software Development, 6:83-110, 2009.

[14] J. Savolainen, N. Niu, T. Mikkonen, and T. Fogdal. Long-term product line sustainability with planned staged investments. IEEE Software, 30(6):63-69, November/December 2013.

[15] G. Trikleris. https://issues.jboss.org/browse/JBTM-2617.

[16] W. Wang, A. Gupta, N. Niu, L. D. Xu, J.-R. C. Cheng, and Z. Niu. Automatically tracing dependability requirements via term-based relevance feedback. IEEE Transactions on Industrial Informatics, 14(1):342-349, January 2018. 